\title{
Plénitude du vide : dévoiement libertin des vanités classiques
}

Hommes-bulles et bulles de Champagne, boutons de roses dans les bosquets et sous les corsets, fastes et falbalas du rococo, musique et romans du jour empilés dans un coin de boudoir avec les masques des soirs de carnavals ; mais aussi, sur les cheminées des petites maisons, sinistres horloges qui scandent la fuite du temps. Ce pourraient être les symboles d'une vanité classique avertissant de détourner son âme de ces frivolités à temps pour l'autre vie. Il s'agit en fait des éléments composant le décor des récits libertins et peignant moins la misère que la félicité du mondain capable de jouissances aussi terrestres qu'évanescentes. Vains divertissements depuis une perspective chrétienne et classique. Mais le superflu, déclare Voltaire, est devenu « chose très nécessaire » $(1961, v .22)$ et les vanités essentielles aux bons vivants de cet âge éclairé.

Cet article propose de lire la fiction libertine du XVIII siècle comme une vanité littéraire puisque s'y exprime, en filigrane, la grande question de la finitude humaine. J'irai même jusqu'à dire que si " chaque époque a eu ses vanités » (Nitti, 2010, p. 54), celles du siècle des Lumières sont peut-être bien à chercher du côté des auteurs dits " libertins ». Qui, en effet, a su ou osé analyser le cœur et l'esprit de leurs contemporains plus finement et plus franchement que ces moralistes se jouant de la censure comme des vieux dogmes idéologiques ? Romans mondains de Crébillon, Duclos ou Laclos, best-sellers pornographiques, contes orientaux de Diderot ou de Voisenon jusqu'aux écrits du marquis de Sade, ces textes ont en commun d'avoir fait du plaisir leur objet principal. Ils ont encore en commun d'avoir perçu que celui-ci servait

- Marine Ganofsky - docteur ès lettres, maître de conférences à l'Université de St Andrews. Adresse pour correspondance : Union Street, University of St Andrews, KY16 9PH, St Andrews, Royaume-Uni ; e-mail : mg216@st-andrews.ac.uk 
souvent à tenir à distance les nouvelles angoisses d'une époque éclairée pour le meilleur et pour le pire. Car en perdant la peur de Dieu et de l'Enfer, le siècle des Lumières perd aussi les promesses qui rassuraient le Chrétien autrefois. Sans éternité pour compenser l'éphémère vie sur terre, le présent seul compte. Ainsi se développe une sagesse hédoniste teintée d’épicurisme et de stoïcisme qui remplace les memento mori de l'âge classique par autant de carpe diem. Toutefois, la fiction libertine, en véritable vanité littéraire, invite ses lecteurs à se demander si cette sagesse axée sur l'illusion du bonheur suffit à écarter la peur de la finitude, si la vanité des plaisirs réussit à éloigner la notion de la vanité de la vie.

La vanité en question ici n'est donc pas celle qui serait synonyme d'orgueil mais celle qui, selon certains, caractériserait la condition humaine. La vie serait vaine parce que vouée au néant de la mort. Étudier le discours et les représentations de cette vanité au siècle des Lumières représente ainsi une invitation formidable à prendre la mesure des métamorphoses affectant aussi radicalement qu'irrévocablement la pensée occidentale à l'aube de l'ère moderne. Se pencher sur la vanité, c'est en effet aborder la question des conceptions du corps et de l'âme : est-il si vil qu'on l'a dit ? Est-elle si immortelle qu'on l'a cru ? C'est également analyser l'expérience du temps à une époque donnée et l'attitude d'une société face à la menace de la mort. C'est aussi se poser la question de la relation à Dieu et aux promesses de la foi. C'est enfin comprendre quel sens était donné à la condition humaine : grandeur ou misère des êtres mortels ? Encore convient-il de se demander comment s'expriment de telles questions en un siècle qui refuse de songer à la mort et, qui plus est, au sein d'une littérature qui serait - en tout cas en surface - un divertissement des plus légers.

Sans tomber dans le piège de la surinterprétation des signes, il s'agira toutefois de prêter attention à la part d'ombre de ces textes de plaisir afin de montrer comment le siècle de la volupté (Goncourt, 1982, p. 145) fait face à la question de la vanité de la vie humaine. Cette approche de la topique libertine s'inscrit dans la lignée des travaux de Robert Mauzi (1965) et de Robert Favre (1978) qui avaient révélé la face obscure de la recherche du bonheur au XVIII siècle, de Michel Delon (2011) qui soulignait la proximité de la mélancolie et du libertinage, ou encore de Jean Starobinski qui notait le côté « danse macabre » de certains bals masqués (1987, p. 85). Se révèleront alors la profondeur des personnages superficiels, la gravité derrière la légèreté, les angoisses sous les rires, et la nécessité de la vanité. Cet oxymore est essentiel pour comprendre le siècle de Mozart, Vivaldi, Boucher, Tiepolo, Beaumarchais, Crébillon, Voltaire, Sterne et Casanova. Leurs œuvres semblent suggérer, comme en contrepoint à L'Ecclésiaste, que sous le soleil, rien n'est vanité : ni la vie humaine, toute infime qu'elle soit, ni même la vanité. Pour de simples mortels, peut-on trouver la plénitude ailleurs que dans le vide des petits-riens ? En soulevant ces questions et en mettant en scène les tentatives de réponses de leurs personnages, les auteurs libertins du siècle des Lumières ont-ils dévoyé, débauché, les motifs des vanités classiques afin d'exprimer une nouvelle conception (née d'une nouvelle angoisse) de la mortalité, du passage du temps ou du sens de la vie ? Ou bien ont-ils plutôt sagement et fidèlement 
conservé l'essence du genre des vanitas en utilisant ce medium pour mettre le lecteur face à la grande question : comment vivre quand on se sait mortel ?

\section{De Memento mori à Carpe diem : oublier la mort au siècle des Lumières}

Tout est vanité, nous dit L'Ecclésiaste, puisque tout doit finir. Tout serait futile face à l'inévitabilité de la mort. Telles sont les révélations plus ou moins furtives de chaque tableau ou texte de vanité. Ce qui est en jeu à chaque fois qu'il est question de vanité, c'est la relation que l'individu entretient avec sa finitude. Or l'idée de mort se transforme radicalement au siècle des Lumières. Avec elle, le genre des vanités prend lui aussi un nouveau visage. Les crânes grimaçants du baroque sont remplacés par les masques souriants du rococo. Mais sous ces masques, les angoisses sur la potentielle vanité de l'existence sont toujours là.

Si les esprits éclairés ne redoutent plus le Jugement dernier à l'heure de leur mort, celle-ci n'en est pas moins terrifiante. La promesse d'une vie éternelle consolait les croyants ; le néant seul désormais attend l'homme ou la femme des Lumières. L'individu éclairé se sait libre de ses vieilles illusions mais aussi, face à la mort, inconsolable de les avoir perdues. La mort ayant été dépouillée des promesses d'éternité qui en rendaient l'idée supportable, on comprend pourquoi, plus que jamais, les hommes et les femmes des Lumières préfèrent tout simplement ne pas y penser. Les vanités du XVIII e siècle devront s'exprimer moins frontalement qu'à l'âge baroque ou à l'heure du gothique. Plus subtiles, ces vanités n'en sont pas moins profondes. Elles posent d'autres questions, moins simples que celle du Bien et du Mal, du paradis et de l'enfer :

L'imprégnation de la pensée européenne par le rationalisme des Lumières sonne le glas de l'âge d'or des vanités en Europe. [...] Les vanités d'aujourd'hui ne répondent plus à une 'stratégie d'intimidation' théologique ; au contraire, elles interrogent souvent, avec ironie ou virulence, le néant, le vide, la déliquescence du sacré. (Quin, 2008, p. 28)

La création littéraire permet de prendre la pleine mesure de l'impact et de la complexité de cette nouvelle donne face à la finitude. Comme l’écrit Michel Delon, « une grande part des fictions du XVIII siècle reste en suspens entre la joie d'en avoir fini avec les mortifications, avec toutes les lisières d'une morale religieuse, et l'inquiétude d'avoir perdu jusqu'aux derniers garde-fous » (2011, p.117). D’un côté, la liberté ; de l'autre, l'angoisse du vide. Or, c'est la littérature libertine, dans sa volupté aux accords mélancoliques, qui va le mieux capturer cette oscillation et repenser par-là le genre des vanités.

Le personnage du libertin ou de la libertine se retrouve en effet au centre de la reconfiguration de la vanité au XVIII ${ }^{e}$ siècle. De cette époque, il incarne à la fois 
les lumières et l'hédonisme qui se rejoignent en une volonté de ne pas laisser la mort future gâter la vie présente. Le roué Saint-Pouange dans L'Ingénu matérialise certes les vices des libertins mais aussi leur stratégie d'adaptation à une nouvelle conscience du néant. En apercevant une bière mortuaire, il « détourne les yeux avec ce simple dégoût d'un homme nourri dans les plaisirs, qui pense qu'on doit lui épargner tout spectacle qui pourrait le ramener à la contemplation de la misère humaine " (Voltaire, 1979, p. 346). Odieux par ailleurs, Saint-Pouange semble ici plus digne de pitié que de mépris. Si faiblesse il y a, ce serait moins face à la tentation des plaisirs que face à l'horreur de la mortalité. La simple idée de la mort peut faire chavirer le mondain dans une spirale mortifère. Loin des memento mori du passé, il faut au contraire oublier que l'on doit mourir. L'art de vivre (pour soi) et le savoir-vivre (pour les autres) exigent qu'on n'en parle pas, qu'on n'y songe pas. La fonction traditionnelle des vanités chrétiennes est mise sens dessus dessous. Autrefois, elles participaient d'un processus de rappel de la mort à venir. Désormais, elles s'articulent autour de l'oubli en une injonction à jouir de la vie ici et maintenant. Ainsi s'explique pourquoi la vanité, ordinairement sujet de prédilection des sermonneurs, se retrouve au cœur d'une littérature du plaisir et de l'incroyance.

\section{Sagesse de l'homme bulle : rien n'est vanité}

Moins frivole qu'il n'y paraît, la littérature dite " libertine » du siècle des Lumières doit se lire comme le fruit de l'indépendance intellectuelle de leurs auteurs et de leurs lecteurs. Ceux-ci sont les descendants directs des libertins « érudits » du Grand Siècle et les frères des philosophes. Leurs écrits témoignent eux aussi d'un rejet absolu des perspectives de Saint Augustin et de Pascal sur la misère de l'homme dont rien sauf l'amour de Dieu ne saurait le tirer. Comme les philosophes, les auteurs de fiction libertine répondent aux Pensées de Pascal pour mieux les démolir et affirmer la grandeur de l'homme en sa finitude ${ }^{1}$. Et comme les libertins érudits, ils proposent une interprétation épicurienne plutôt que chrétienne de L'Ecclésiaste. Jean-Charles Darmon a étudié les sources classiques derrière la lecture non-conventionnelle qu'en ont faite Chaulieu ou Des Barreaux. Dans leurs œuvres, explique-t-il, le «'tout est vanité' se conjugue avec un 'hâte-toi de jouir avant qu'il ne soit trop tard' inspiré d'Horace et d'Anacréon " (Darmon, 2011, p. 63). Nos auteurs de littérature voluptueuse et éclairée au siècle des Lumières ont les mêmes idoles : Anacréon leur donne toujours des leçons de légèreté, Horace est toujours, avec Ovide, le maître d'un art de jouir et de vivre au jour le jour, Epicure et Lucrèce inspirent encore leur vision matérialiste de l'homme voué à se rendre heureux et impuissant face à l'entropie. La libération des mœurs avec la Régence permet à ces théories d'être mises en pratique par un grand nombre de bons vivants que la libération des idées ne contraint plus

1. Sur Voltaire et Pascal, voir Métayer (2011). 
(ou moins) à la clandestinité. Un tourbillon de plaisirs emporte la société française à la mort du Roi Soleil et les idées des philosophes ont le vent en poupe. Pourtant, sous la surface badine de la Régence et du début de règne de Louis XV, les vanités ont pris un tour plus grave. C'est que les illusions et les consolations d'autrefois se sont véritablement dissoutes dans la lucidité de cette ère nouvelle. Se met alors en place un véritable " art de l'éloignement" (Darmon, 2011, p. 97) dont l'enjeu est de tenir les angoisses à distance, à défaut de pouvoir les supprimer. Avec Watteau les fêtes galantes se détachent de fonds crépusculaires, chez Mozart les notes s'envolent au-dessus de sombres accords, et dans la littérature libertine les bons vivants courent d'un plaisir à l'autre pour mieux oublier l'image d'un linceul. La sagesse de ces hommesbulles est d'avoir compris que le bonheur dépendait souvent d'une illusion de légèreté.

Ironiquement, pour les esprits éclairés, les vieilles illusions de la foi laissent un vide que peut seule combler une autre illusion : celle du divertissement. Le divertissement, si décrié par Pascal, devient crucial dans cette quête d'oubli, et ce précisément pour les raisons énoncées par l'auteur des Pensées : parce que l'homme ne peut supporter le vide et le silence de sa chambre la nuit; parce qu'il s'agit bel et bien de divertir (au sens premier de « détourner ») l'esprit de ce qui lui pèse. Divertissement donc moins vain qu'il n'y paraît s'il remplit sa fonction de mécanisme d'adaptation à une condition humaine devenue insupportable. Pascal et Saint Augustin, l'Église et ses dévots mettaient leur public en garde contre les illusions vaniteuses des plaisirs d'ici-bas. La vraie vie commencerait après la mort, dans l'amour de Dieu. Vanitas s'oppose chez eux à Veritas. Pourtant chez nos libertins, comme d'ailleurs chez Montaigne (voir Compagnon, 2011, p. 26), Vanitas (le vide, la fragilité de l'existence) se confronte plutôt à Voluptas (le plaisir qui, comblant ce vide, fait oublier cette fragilité). Le plaisir, loin d'être une vanité (une inanité) de plus, devient essentiel pour ces êtres des Lumières puisqu'il est d'une part un divertissement et d'autre part une sensation. Or, la sensation, en ce siècle bercé de philosophie sensualiste et matérialiste, est ce par quoi l'être peut reconquérir le sentiment de son existence : "Je sens donc je suis", découvre la statue de Condillac (1754) avec les grands sensuels de cet âge voluptueux. Cette nouvelle conception de l'être et du néant est l'une des conséquences de la remise en question des repères chrétiens et de l'ascension des théories néo-épicuriennes sur la matière et ses sensations, explique Georges Poulet : "puisque ce néant est insensibilité pure, échapper au néant, ce sera éprouver des sensations» $(1949$, p. 27). Ainsi, les vanités libertines au XVIII siècle opposent au vide de l'apathie la plénitude (quoiqu'évanescente) des sensations. Le temps d'un instant, Eros vainc Thanatos. Les libertins, toujours rebelles aux limites, affronteraient même celle de la mort. Leur pire ennemie (pensons à Casanova qui déclarait " détester ", ce "monstre » dans la préface de ses mémoires - 2013, p. 14) ne semble plus si invincible face à l'intensité du plaisir qui compense l'éternité perdue.

Cette revalorisation néo-épicurienne de la sensation va main dans la main avec une revalorisation de la chair qui re-polarise absolument la tradition des vanités. 
Loin du dualisme augustinien, le corps sensible ne signifie plus la misère de la condition humaine mais au contraire sa grandeur. Sur les tableaux et dans les textes du siècle de Diderot et de Fragonard, les crânes des vanités baroques ou les squelettes des danses macabres sont détrônés par les chairs érubescentes et rebondies des personnages. C'est par le corps vivant que l'individu peut parvenir à toucher du doigt la seule plénitude accessible aux mortels et surtout accomplir sa véritable destinée : non pas celle de la putréfaction de la chair mais celle du bonheur vers lequel nos appétits nous conduisent. L'Ecclésiaste est pris au mot par les libertins : "Si son âme ne s'est point rassasiée de bonheur [...] il est venu en vain » (Ecc. 6.4). Le texte libertin le plus didactique de son époque, Thérèse philosophe (1754), le déclare solennellement : l'homme a été créé (par Dieu ou par la Nature selon la métaphysique de chacun) comme un être d'appétits afin de survivre et de propager son espèce. Les expressions « vain/e/s » ou "vanité/s » sont uniquement utilisées dans ce texte pour qualifier la répression de ces désirs (et non ces désirs eux-mêmes). C’est la leçon encore de ces autres textes philosophico-pornographiques comme La Philosophie dans le boudoir du marquis de Sade (1795), de L'Education de Laure (1786, anonyme) mais aussi des écrits de Diderot et de ses compères matérialistes d'Holbach, La Mettrie ou Helvétius. Contre la gageure de Pascal, les libertins du siècle des Lumières misent tout sur cette vie-là. Elle est après tout, dans leur univers métaphysique, la seule certitude possible, outre celle de la mort.

Car la mort viendra bien assez tôt. S'ils veulent l'oublier, les libertins ne l'ignorent pas. Pour ces sages, le caractère éphémère de l'existence, loin de la rendre "vaine ", ne la rend que plus précieuse : «la connaissance des limites du temps humain ne doit pas conduire à désespérer, mais à mettre ce temps à profit ", remarque Karine Lanini à propos de la pensée de Sénèque dont se souviendront les libertins du Grand Siècle et ceux des Lumières (2006, p. 188-189). Dans l'univers libertin des petites maisons et des boudoirs, l'incitation au plaisir sous la menace de la clepsydre est plus discrète mais non moins palpable que dans les modèles antiques. Nul besoin de la larva, ce squelette articulé que faisait amener le sensuel Trimalcion pour inciter ses convives à s'abandonner aux délectations de son festin : «Vivons donc, tant que l'âge à jouir nous convie " (Pétrone, 1923, p. 131). L'homme et la femme éclairés lisent de telles injonctions dans la simple présence d'horloges dans les salons : " mode lugubre. Il n'y a rien de si triste à contempler qu'une pendule : vous voyez votre vie s'écouler » (Mercier, 1944, t. ii, p. 1571). Il n'est pas anodin que parmi les chefs d'œuvre de l'écriture libertine se trouvent des titres comme Point de lendemain de Denon (1777) et La Nuit et le moment de Crébillon (1755). Il y a, dans la fiction libertine, une anxiété palpable vis-à-vis du passage du temps qui se manifeste à la fois dans la volonté des personnages de suspendre le temps au moment du plaisir et dans leur urgence de jouir, de passer rapidement, sans temps mort, d'un plaisir l'autre.

Il y a bel et bien urgence pour ces bons vivants qui savent qu'avant la mort, la vieillesse viendra mettre un terme à leurs réjouissances. Une autre leçon qu'ils ont retenue de L'Ecclésiaste est celle qu'il y a un temps pour tout : " Au jour du bonheur, sois 
heureux, et au jour du malheur, réfléchis » (Ecc. 7.14). Les vanités traditionnelles mettaient en porte-à-faux vita voluptuaria, vita practica et vita contemplativa (voir Tapié, 1991, p. 200). Les vanités de la fiction libertine présentent ces trois « vies » non comme des alternatives mutuellement exclusives mais comme des étapes successives au sein d'une seule et même existence. Le temps fuit, la jeunesse passe : carpe diem. Peu ou prou de vieillards dans la littérature libertine; c'est une fiction de la jeunesse consciente qu'elle doit vivre vite parce que les plaisirs ne dureront qu'un temps, et légèrement parce que la gravité viendra bien assez tôt avec l'âge.

Ainsi s'exprime au XVIII siècle la sagesse de l'homo bulla : papillon de cour ou belle évaporée, frivole marquis ou fille légère. C'est une sagesse de l'illusion. La légèreté est un effort d'oubli des angoisses que provoque une condition humaine révélée comme inexorablement vouée au néant. Cette sagesse leur permet de trouver la plénitude dans le vide des petits riens, des petits plaisirs. La plénitude, pour un être sans Dieu ni paradis, ne se trouve plus nulle part ailleurs. Ainsi, c'est la notion de vanité même que la sagesse libertine remet en question : rien n'est vanité, pas même la vanité si celle-ci parvient à tenir à distance l'angoisse du vide. Les sages hédonistes ne sauraient être vains en vain.

\section{Des vanités littéraires et libertines : le lecteur en son miroir}

Toutefois, on aurait tort de ne voir dans la fiction libertine qu'une littérature sapientale dont l'objet serait de faire l'apologie du libertinage. Ce qui fait de cet ensemble de textes (des plus hétéroclite certes mais néanmoins cohérent dans sa conjonction de lumières et d'érotisme) une véritable vanité littéraire, c'est justement son ouverture sur un choix offert au lecteur. Bien que l'option de la légèreté lui soit présentée à travers les aventures des personnages, l'impéritie de cette option est elle aussi suggérée. Ces textes de plaisir recèlent dans leur ombre une indécision entre la peur du néant et l'effort pour l'oublier.

L'essence des vanités picturales ou littéraires est ce choix face auquel l'œuvre place son public une fois rappelée la nature éphémère de l'existence : "Ce n'est donc pas tant une série de motifs symboliques qui importent (le crâne, la bougie ou la bulle) que la postulation de leur dépassement ou de leur contournement. Toute vanité figure un choix, un 'ou bien' en proposant toujours au destinataire au moins deux possibilités de lecture " (Laborie, 2012, p. 3). Or, à l'aube des Lumières, la nature de ce choix se transforme. Dans le modèle religieux des vanités, le choix est binaire : d'un côté, les plaisirs de ce monde, illusoires (d'où tous ces miroirs) et évanescents, toujours déjà en déliquescence; d'un autre côté, la suggestion d'une béatitude réelle et éternelle dans l'amour de Dieu. Cependant, à partir du XVII siècle déjà comme en témoignent certaines vanités baroques (voir Lanini, 2006, p. 16), l'option de Dieu étant de plus en plus mise en doute, le dilemme évoqué par les vanités est tout autre. Le lecteur est mis face à un nouveau choix, moins binaire, moins facile que celui 
des modèles religieux. D'un côté, toujours les mêmes plaisirs, toujours aussi «vains " (frivoles). Mais de l'autre côté, l'alternative n'est plus la béatitude du saint : c'est l'angoisse de l'Homme qui se sait seul face à son destin et confronte la vanité (la fragilité) de son existence. Ainsi l'alternative du dépassement dont parle Laborie ne concerne plus le dépassement de la vanité humaine dans la foi en la vie éternelle. Elle concerne, avec les libertins, le dépassement de l'angoisse dans les plaisirs. Le choix existentiel qui caractérise les vanités artistiques est toujours là, mais ses options sont limitées : l'angoisse du néant d'un côté, et de l'autre l'oubli de cette angoisse dans les plaisirs.

Signe du temps et de ses doutes, les narrations libertines n'offrent pas de réponse claire, pas de guide évident. Le lecteur doit cheminer seul. Nous sommes au temps des Lumières et donc du doute méthodique. Les philosophes, et avec eux les auteurs libertins, exigent un questionnement actif de la part de leurs lecteurs. D'où les dialogues de Diderot, les mystères des contes de Voltaire, mais aussi les fins ouvertes de Denon, Crébillon, La Morlière ou Laclos. D'où surtout l'ironie de leurs narrations qui fait osciller leurs récits entre burlesque et sérieux, entre éloge et critique du libertinage. Or, cette écriture caractéristique du style libertin est aussi l'essence du genre littéraire des vanités en ce qu'elle ouvre sur le doute : "l'ambiguité et l'indécision du destinataire, après le constat de l'inconsistance du monde et des plaisirs, la mise en scène de l'hésitation interprétative constituant, en effet, le premier critère d'une vanité littéraire [...]. L'exploration de l'entre-deux, les figures de retournement, le narrateur indécis, la modalité paradoxale, parodique et/ou ironique proposent autant de traitements distanciés du thème " (Laborie, 2012, p. 13-14). L'ironie dans la fiction libertine se trouve plus que tout dans l'intromission d'un doute quant à l'efficacité de la recherche effrénée de plaisirs sensuels et éphémères. En effet, à y regarder de près, ces textes apparemment légers sont ponctués de graves réflexions sur la condition non pas simplement sociale, nationale, sexuelle etc. mais aussi humaine de leurs personnages. Il n'est pas rare que des réflexions sur la mort ou des allusions à la peur du vide (ennui, solitude, silence) ouvrent le récit de plaisir, comme pour suggérer au lecteur que ce plaisir peut être une réaction plus ou moins consciente contre l'angoisse qui travaille les papillons mondains. Le récit du Sopha est présenté comme un rempart contre l'ennui. Les premières pages de Thémidore multiplient les allusions au temps qui passe ; le joyeux roman Les Sonnettes s'ouvre sur la mort et la maladie des parents du héros. Son futur libertinage pourra alors être présenté comme une sage réaction face à cette conscience de la fragilité de l'existence. Toutefois, le lecteur ne trouve dans ces textes ni condamnation ouverte ni apologie sincère ni pathos sentimental; ni claire admiration ni moquerie cruelle : rien que les possibilités d'interprétation ouvertes par l'ironie mélancolique du narrateur. Car ces auteurs de récits érotiques parlent de libertinage sans qu'il y ait nécessairement de complaisance de leur part pour leur objet. Si l'on peut certes déceler dans leur prose une fascination pour la très bonne société qu'ils fantasment comme étant aussi voluptueuse quélégante dans ses vices (voir Rustin, 1978, p. 27), c'est surtout l'ironie qui domine leurs narrations. Le sens est en suspens dans cet espace ouvert par l'ironie 
qui met le lecteur face à la responsabilité de décider par lui-même comment interpréter ces plaisirs. En refusant de répondre au doute qu'il rend palpable par l'ouverture interprétative de l'ironie, le texte libertin pose au lecteur la même question que les natures mortes baroques ou les monuments funéraires médiévaux : toi, te sachant mortel, quelle voie choisis-tu?

Pour forcer ses lecteurs à contempler ce choix, la littérature libertine a recours aux mêmes techniques que les vanités traditionnelles. Comme dans les natures mortes baroques, le lecteur/spectateur est sollicité dans sa chair même. L'on veut croquer le fruit si mûr peint sur la toile quand bien même celle-ci suggère la vanité de ce désir. Pareillement, le récit érotique déclenche chez son lecteur des réactions somatiques, un appétit bien réel, alors même que le texte suggère la mélancolie des personnages pris dans une frénésie de plaisirs. Ainsi touché, le lecteur/spectateur devient sujet plutôt que simple témoin du choix posé par l'œuvre de vanité : "L'esthétisation est donc la condition de la réception du discours de la vanité. Il s'agit de susciter des émotions esthétiques en sollicitant les sens et les puissances de l'âme " (Brunel, 2012, p. 7). Alors, l'œuvre de vanité peut jouer pleinement son rôle de miroir : « Le véritable objet des vanités, c'est nous [...] les regardeurs, les vivants » (Wajcman, 2010, p. 117). C'est en ce sens enfin que la littérature libertine se révèle être véritablement une vanité littéraire : elle offre au lecteur un miroir dans lequel il contemple sa propre condition humaine. Les plaisirs voire les vices des personnages libertins peuvent ne pas être les nôtres, mais, entre deux plaisirs, leur intuition des limites humaines ne nous touche pas moins. Nous mettre face à notre propre vanité (notre fragilité) est la technique de base des vanités traditionnelles : le Moyen-Âge optait pour les squelettes des danses macabres, l'âge baroque pour des crânes qui sont toujours une vision de notre état futur; dans la fiction libertine du XVIII ${ }^{e}$ siècle, c'est la peur $\mathrm{du}$ vide qui fait vibrer une corde sensible atemporelle et universelle. Ainsi, ni tout à fait sujet de dérision ni tout à fait objet d'admiration, le personnage du libertin pourrait bien être en fait la plus proche et la plus honnête anamorphose de nousmêmes. Au miroir déformant tendu par la fiction libertine se reflète l'humanité dans toute sa fragilité mais aussi toute sa grandeur : une humanité capable, par les illusions du plaisir, de s'émanciper de sa misère le temps d'un instant.

Nul besoin de crânes, de sabliers ni de fleurs fanées pour faire de la fiction libertine du XVIII siècle une vanité littéraire. Il lui suffit de mettre ses lecteurs face au doute qui enveloppe désormais, pour l'homme et la femme éclairés et déjà modernes, la question du comment vivre face à la menace du néant. La légèreté des personnages libertins apparait comme un mécanisme d'adaptation à une condition humaine que la perte des dernières consolations métaphysiques révélait dans toute sa finitude. Si tout est vanité, rien n'est vanité : tout ce qui peut aider à supporter ou oublier ses angoisses existentielles ne saurait être recherché en vain. Cependant, le filigrane de ces textes du libertinage pose la question de l'efficacité d'un art de vivre tout en légèreté. $\mathrm{Au}$ lieu d'y trouver le réconfort d'une sagesse à toute épreuve, le lecteur attentif y découvre le reflet de ses propres inquiétudes. L'ironie avec laquelle s'écrit le libertinage 
au XVIII ${ }^{e}$ siècle ne prête pas qu'à sourire. Dans la distance qu'elle établit entre l'objet libertin et son lecteur, l'ironie introduit le doute. Elle insinue la mélancolie. Comme dans les vanités baroques et les memento mori d'antan, la fiction libertine figure le vide d'une part et d'autre part du choix existentiel : comment vivre face à la vanité de la vie humaine ? Comme dans ces modèles, tout effort semble bien vain. À moins qu'on ne sache se contenter de l'illusion d'un moment, jouir de la plénitude du vide.

\section{RÉFÉRENCES}

Brunel, T. (2012). «Vanités textuelles ", «Vanités littéraires », validité du concept et critères de reconnaissance dans la littérature du XVII siècle ? Études Épistémè, 22. Récupéré de https://journals.openedition.org/episteme/365

Casanova, G. (2013). Histoire de ma vie [1789-1798]. G. Lahouati et M.-F. Luna (éds.), Paris, France : Gallimard.

Compagnon, A. (2011). "Vaines pointures, mais toujours pointures ": Montaigne et L'Ecclésiaste. Dans J.-C. Darmon (dir.), Littérature et vanité. La trace de "L'Ecclésiaste » de Montaigne aux temps présents (p. 9-27). Paris, France : Presses Universitaires Françaises.

Darmon, J.-C. (2011). Un Ecclésiaste libertin ? Dans J.-C. Darmon (dir.), Littérature et vanité. La trace de "L'Ecclésiaste", de Montaigne aux temps présents (p. 61-98). Paris, France : Presses Universitaires Françaises.

Delon, M. (2011). Le Principe de délicatesse. Libertinage et mélancolie au XVIII siècle. Paris, France : Albin Michel.

Favre, R. (1978). La Mort dans la littérature et la pensée françaises au siècle des Lumières. Lyon, France : Presses Universitaires de Lyon.

Goncourt, E. et J. de. (1982). La Femme au XVIII siècle [1862]. Paris, France : Flammarion.

Klibansky, R., E. Panofsky et F. Saxl (1964). Saturn and Melancholy: Studies in the History of Natural Philosophy, Religion and Art. Londres, Royaume Uni : Nelson.

Laborie, J.-C. (2012). Vanités d'hier et d'aujourd'hui : permanence de l'éphémère. Études Épistémè, 22. Récupéré de https://journals.openedition.org/episteme/359

Lanini, K. (2006). Dire la vanité à l'âge classique. Paradoxes d'un discours. Paris, France: Champion.

Mauzi, R. (1965). L'Idée du bonheur dans la littérature et la pensée françaises au XVIII siècle. Paris, France : Slatkine.

Mercier, L.-S. (1944). Tableau de Paris [1781]. Paris, France : Mercure de France.

Métayer, G. (2011). Un Ecclésiaste voltairien ? Dans J.-C. Darmon (dir.), Littérature et vanité. La trace de "L'Ecclésiaste » de Montaigne aux temps présents (p. 99-118). Paris, France : Presses Universitaires Françaises.

Nitti, P. (dir.) (2010). C’est la vie! Vanités de Pompéi à Damien Hirst. Paris, France : ESFP.

Pétrone. (1923). L'Euvre de Pétrone : le Satyricon (Ier siècle après J.-C.). (L. de Langle trad.). Paris, France : Bibliothèque des curieux.

Poulet, G. (1949). Études sur le temps humain. T. $1:$ La durée intérieure. Paris, France : Plon.

Quin, E. (dir.) (2008). Le Livre des vanités. Paris, France : Regard.

Rustin, J. (1978), Définition et explicitation du roman libertin au siècle des Lumières. Travaux de linguistique et de littérature, 16, 27-34.

Starobinski, J. (1987). L'Invention de la liberté : 1700-1789. Genève, Suisse : Skira. 
Tapié, A. (dir.) (1991). Les Vanités dans la peinture au XVII siècle. Méditations sur la richesse, le dénuement et la rédemption. Paris, France : Réunion des Musées Nationaux.

Tapié, A. (2010). Vanité. Mort, que me veux-tu ? Paris, France : La Martinière.

Voltaire. (1961). 'Le mondain' [1736]. Dans J. Van Den Heuvel (éd.), Voltaire : Mélanges (p. 203-206). Paris, France : Gallimard.

Voltaire. (1979). L'Ingénu [1767]. Dans F. Deloffre et J. Van Den Heuvel (éds.), Voltaire : Romans et contes (p. 285-347). Paris, France : Gallimard.

Vovelle, M. (1983). La Mort et l'Occident de 1300 à nos jours. Paris, France : Gallimard.

Wajcman, G. (2010). El Embalsamador. Méditation sur les vanités, l'image et les quatre morts.

Dans P. Nitti (dir.), C’est la vie! Vanités de Pompéi à Damien Hirst (p. 117-19). Paris, France : ESFP.

RÉSUMÉ : Les Vanités composeraient-elles l'essence trop souvent oubliée de la littérature dite "libertine " du dix-huitième siècle ? La fiction voluptueuse du siècle des Lumières a observé et problématisé la nouvelle condition humaine à l'aube de la modernité : plus de Dieu capable de remplir le vide ; plus d'éternité à espérer au-delà de la finitude humaine ; plus rien que l'humain, le moment présent et la vérité de la sensation. Les personnages de cette littérature érotique mettent en scène une sagesse de l'homme-bulle qui serait un mécanisme d'adaptation à cette nouvelle réalité. Rien n'est vain pour ces êtres légers, tant que la vanité des plaisirs tient à distance le souvenir de la vanité de l'existence que menace le néant. Cependant, l'ironie de ces narrations libertines suggère, comme dans le modèle classique, que ces jouissances temporelles peuvent ne pas suffire à combler l'angoisse du vide. Une question est posée au lecteur qui surprend, dans la figure du libertin, l'anamorphose de l'humain : toi, te sachant mortel, entre angoisse et insouciance, quelle voie choisis-tu?

Mots-clés : libertinage, Lumières, mort, ironie, XVIII ${ }^{\mathrm{e}}$ siècle

\section{Finding plenitude in a void. The libertine subversion of classical vanities}

ABSTRACT: Could vanities be the overlooked essence of so-called 'libertine' fiction? $18^{\text {th }}$ century French erotic literature has observed and analysed the new human condition on the brink of modernity. There is no more God to fill up the void, no more eternity to hope for beyond human finitude; only humanity, the present moment and the truth of a sensation. The adventures of this fiction's characters stage the wisdom of the homo bulla that would be, tacitly, a coping mechanism to that new reality. Nothing is vanity for these frivolous beings, as long as the vanity of pleasures manages to keep away the memory of the vanity of life. However, the irony of those narrations suggests, like in the classical model of vanitas, that these temporal delectations may not be enough to avoid angst. The reader is asked a question as he or she discovers in the libertine figure an anamorphosis of all humanity: knowing that you are mortal, what will you choose between anguishes and vanities?

Keywords: Libertines, enlightenment, death, irony, eighteenth century 